\title{
Stroke Recurrence in First-Ever Symptomatic Carotid Web: A Cohort Study
}

\author{
Stephane Olindo, ${ }^{\mathrm{a}}$ Nicolas Chausson, ${ }^{\mathrm{b}}$ Aissatou Signate, ${ }^{\mathrm{c}}$ Sylvie Mecharles, ${ }^{\mathrm{d}}$ Jean-Luc Hennequin, ${ }^{\mathrm{e}}$ \\ Martine Saint-Vil, ${ }^{\mathrm{c}}$ Mireille Edimonana-Kaptue, ${ }^{\mathrm{c}}$ Severine Jeannin, ${ }^{\mathrm{c}}$ Anne Landais, ${ }^{\mathrm{d}}$ Philippe Cabre, ${ }^{\mathrm{c}}$ \\ Igor Sibon, ${ }^{\mathrm{a}}$ Didier Smadja, ${ }^{\mathrm{b}}$ Julien Joux ${ }^{\mathrm{c}}$ \\ ${ }^{\text {aS }}$ troke Unit, Bordeaux University Hospital, Bordeaux, France \\ ${ }^{b}$ Neurology Department, Sud-Francilien Hospital, Corbeil-Essonnes, France \\ 'Neurology Department, Martinique University Hospital, Martinique \\ ${ }^{d}$ Neurology Department, Guadeloupe University Hospital, Guadeloupe \\ 'Vascular Surgery Department, Martinique University Hospital, Martinique
}

Background and Purpose Carotid web (CaW) is an intimal variant of fibromuscular dysplasia responsible for ipsilateral cerebral ischemic events (CIE). Symptomatic CaW likely has a high risk of recurrent $\mathrm{CIE}$, but no salient prospective data are available. We aimed to assess recurrence rate and its predictors after a first-ever CIE.

Methods Consecutive Afro-Caribbean patients who had cryptogenic first-ever CIEs (ischemic stroke [IS] or transient ischemic attack [TIA]) associated with ipsilateral CaW were included in this multicenter observational cohort study. The follow-up (January 2008 to March 2019) focused on CIE recurrences. Kaplan-Meier method assessed rates of recurrences and Cox proportional hazards regression analyzed risk factors.

Results Ninety-two patients (79 first-ever ISs and 13 TIAs; mean age \pm standard deviation, $49.8 \pm 9.9$ years; 52 [56.5\%] women) were included. During a mean follow-up of $50.5 \pm 29.6$ months, 19 (20.7\%) patients experienced recurrent ipsilateral CIEs (16 ISs and three TIAs). Of 23 patients receiving surgery/stenting treatment, no recurrence occurred after the intervention (median follow-up, 39.8 months [interquartile range, 27.6 to 72.4]). Under medical treatment alone, the annual recurrent CIE rate was 6.9\%, and the cumulative rate was $4.4 \%$ at 30-day, $10.8 \%$ at 1-year, 19.8\% at 2-year, 23.2\% at 3-year, and 27.3\% at 5-year. Presence of silent cerebral infarctions was the only independent risk factor of CIE recurrences (hazard ratio, 6.99; 95\% confidence interval, 2.4 to 20.4; $P=0.004$ ).

Conclusions Under medical treatment alone, symptomatic CaW was associated with a high rate of recurrence that reached $27.3 \%$ at 5-year. Surgery/stenting seems to be efficient, and randomized control trials are required to confirm the benefit of these interventions.

Keywords Ischemic stroke; Cohort studies; Carotid artery; Recurrence; Carribbean region; Fibromuscular dysplasia
Correspondence: Stephane Olindo Stroke Unit Department, University Hospital of Bordeaux, Place Amelie Raba-Leon, 33076 Bordeaux Cedex, France

Tel: +33557821263

Fax: +33556798702

E-mail: stephane.olindo@chubordeaux.fr

https://orcid.org/0000-0001-67108153

Received: December 31, 2020

Revised: April 3, 2021

Accepted: April 5, 2021 


\section{Introduction}

Carotid web (CaW) is an intimal and non-circumferential variant of fibromuscular dysplasia located at the origin of the carotid bulb and characterized by a membrane-like lesion protruding into the artery lumen. ${ }^{1-3} \mathrm{CaW}$ is increasingly recognized as a potential etiology of cryptogenic ischemic stroke (CIS) and usually described in middle-age patients with a slight female prevalence. ${ }^{4-7}$ Predominance of $\mathrm{CaW}$ in the black population has been suggested through case series, ${ }_{1,8}^{3,8}$ and a recent systematic literature review showed that African descendants accounted for the largest part (58.6\%) of all reported symptomatic CaW cases. ${ }^{9}$ The widely accepted mechanism of cerebral ischemic events (CIE) is an artery-to-artery embolism through blood stasis rostral to CaW, thrombus formation and clot fragmentation scattered by the blood flow. ${ }^{10}$ Large ischemic strokes (ISs) are often observed and related to a high rate of vessel occlusion. ${ }^{11,12}$ According to single case observations and small case series, symptomatic CaW seems to be associated with a high risk of CIE recurrences ranging from 25\% to $71 \%$ despite anti-thrombotic therapies. ${ }^{3,11,13}$ Although seldom reliable data is available regarding the risk of recurrence, a growing number of CaW patients have been treated with invasive treatment such as surgery or stenting. ${ }^{3,11}$

The aim of the present study was to assess the long-term recurrence risk and its predictors in a large Afro-Caribbean cohort who experienced a first-ever CIE associated with an ipsilateral CaW. Clinical and imaging data of the cohort are also detailed.

\section{Methods}

\section{Study and patients}

In January 2008, this cohort study was implemented to prospectively enroll consecutive Afro-Caribbean patients who had symptomatic CaW admitted to two French Caribbean hospitals, Martinique and Guadeloupe. This study focused only on Afro-Caribbean patients because this ethnic group constitutes the overwhelming majority of the population in these areas and is particularly affected by CaW. The Afro-Caribbean origin was based on self-identification. The University hospital of Martinique was the coordinating center. In 2014, two additional French hospitals, Bordeaux and Corbeil-Essonnes, took part in the constitution of the cohort. Centers were selected according to the clinical experience of local physicians in CaW diagnosis. Patients were included between January 2008 and December 2016 and were followed-up until March 2019. The cohort was observational and each center applied their own therapeutic strategy including invasive preventive treatment such as surgery or stenting. Local ethic committees with the backing of Commission National de l'Informatique et des Libertés (CNIL), the French data protection authority, approved the study.

Patients were included without any age criteria. Symptomatic CaW was defined as CaW in the internal carotid artery, leading to CIE (IS or transient ischemic attack [TIA]) in the carotid territory ipsilateral to the CaW lesion. Patients or next of kin were systematically asked to participate in the cohort study that resulted in periodic consultations at the hospital and by phone call. At the enrollment, the investigators completed a data form that included demographic data, vascular risk factors, patient's medical histories, admission clinical symptoms and the National Institutes of Health Stroke Scale (NIHSS) score, acute and secondary prevention treatments, infarction territory, diffusion-weighted imaging Alberta Stroke Program Early CT Score (DWI-ASPECTS) ${ }^{14}$ in case of middle cerebral artery (MCA) territory infarction, presence of silent cerebral infarction $(\mathrm{SCI})$, presence of artery occlusion and its location. Extra and intracranial arteries were systematically assessed on computed tomography angiography (CTA). SCl was defined as chronic cerebral infarction on imaging without a history of clinical stroke or TIA attributable to the lesion. ${ }^{15}$ For the current study, only patients with first-ever CIE classified as cryptogenic were considered. Cryptogenic IS or TIA was determined after extensive standardized work-up that included intracranial and extracranial vascular imaging with CTA, transthoracic and transesophageal echocardiography, cardiac telemetry monitoring ( $>48$ hours), continuous ambulatory electrocardiography (24 to 72 hours), extensive blood laboratory testing and cerebrospinal fluid analysis when indicated.

\section{Outcome assessment}

The primary outcome was recurrent CIE. Visit at the study sites with face-to-face assessment was performed at 3-month then every 12 months to identify CIE recurrence. For patients who had moved away from the study area, the evaluation was investigated via phone call. When the recurrent CIE was not managed in the investigator's stroke units, the general practitioner was contacted, and the hospitalization report and imaging were analyzed to confirm and classify the event. Information on medical treatment at the time of recurrent CIE was collected. For each recurrent $\mathrm{CIE}$, we determined the etiologic mechanism. Recurrent CIE included IS and TIA. IS was defined as a new focal neurological deficit lasting $>24$ hours or as an acute cerebral infarction on magnetic resonance imaging (MRI) or computed tomography (CT) consistent with the clinical symptoms. ${ }^{15} \mathrm{TIA}$ 
was defined as a new neurological deficit consistent with a transient focal brain dysfunction lasting $<24$ hours without any cerebral infarction on imaging. Fluctuations or worsening of the index stroke were not determined as recurrent CIE. Clinical worsening in relation with brain edema, hemorrhagic transformation of index IS, metabolic and systemic disturbance were not considered as recurrence.

The secondary outcome was the functional status assessed with the modified Rankin Scale (mRS) score 3 months after the first-ever CIE and at the end of follow-up. A favorable outcome was defined as $m R S$ score $\leq 2$.

\section{Case ascertainment of carotid web}

Diagnosis of CaW was performed on CTA that was chosen for its widespread availability, the ability of image reconstruction and the capacity to identify differential pathologies such as atherosclerosis or dissection. CTA is now considered as the method of choice for CaW diagnosis. ${ }^{6,16}$ Carotid artery bulb was analyzed in the axial, coronal and sagittal plane of CTA using a maximal intensity projection reconstruction (Supplementary Figure 1). CTA patterns consistent with CaW were: (1) A linear defect that usually splits the arterial lumen on axial plane. (2) A regular and thin shelf-like intraluminal-filling defect usually arising from the posterior wall of the proximal carotid bulb. (3) The absence of atherosclerosis plaque features such as calcifications or irregularities of the lesion shape. Small protruding lesions ${ }^{13}$ were not categorized as symptomatic CaW. The contralateral carotid bulb was examined and lesions of $\mathrm{CaW}$ were collected. Three investigators (S.O., A.S., and J..J.) systematically analyzed each suspected case and the diagnosis of symptomatic CaW was adjudicated by consensus. Carotid bulb stenosis resulting from CaW protrusion was measured according to the European Carotid Surgery Trial (ECST) method ${ }^{17}$ and was expressed as a percentage.

\section{Statistical analysis}

Categorical variables are presented as number (\%), and continuous variables are expressed as mean \pm standard deviation (SD) or as median with interquartile range (IQR) if skewed. Categorical variables were compared with the chi-square test, and continuous variables were compared with Student's t-test or Mann-Whitney's U test.

The annual recurrent rate was calculated using event numbers and median follow-up time and represents the average number of CIE per 100 person-years. Kaplan-Meier curves were used to estimate the cumulative incidence of CIE or IS recurrence over time. End of follow-up was defined by the day of last visit assessment including patients who were lost to follow-up.
Two analyses were performed: (1) for the whole follow-up period and (2) for the follow-up period under medical treatment alone: for patients who did not receive surgery or stenting, the whole follow-up period was considered; for patients treated with surgery or stenting, the period from the inclusion to the day before surgery or stenting was considered. We used Cox proportional hazards regression analysis to explore risk factors of $\mathrm{CIE}$ recurrence during the follow-up period for patients under medical treatment. A multivariable analysis including factors that had $P$-values of $<0.05$ in univariable analysis was also performed. Results were expressed as hazard ratio (HR) and 95\% confidence interval $(\mathrm{Cl})$. Analyses were performed with StatView 5.0 (Informer Technologies Inc., Roseau Valley, Dominica) and Microsoft Excel software. The level of statistical significance for the $P$-value was set at less than 0.05 .

\section{Results}

Between 2008 January and December 2016, 105 Afro-Caribbean patients were included in our cohort. In the present study, after excluding 13 patients with recurrent CIEs, 92 patients with first-ever CIE were included. Among the 92 patients, 82 were recruited from Martinique, four from Guadeloupe, four from Corbeil-Essonnes, and two from Bordeaux. The median follow-up duration (inclusion to last visit) was 45.5 months (IQR, 32.4 to 68.4). On March 2019, four patients (4.3\%) were lost to follow-up. The demographic, clinical, and imaging baseline data for the 92 symptomatic CaW patients are summarized in Table 1.

\section{Clinical pattern and functional outcome of first-ever CIE associated with a CaW}

The mean age at first-ever CIE was $49.3 \pm 9.7$ years (range, 26 to 72) and $56.5 \%$ were females. Of the included patients, $25.0 \%$ were aged $>55$ years, and $56.5 \%$ did not have any vascular risk factors. The most common risk factors were high blood pressure (20.6\%) and smoking (20.6\%). Mean admission NIHSS score was $7.7 \pm 7.4$ (range, 0 to 27 ), $51.1 \%$ experienced a TIA or a minor stroke (NIHSS <5), 32.6\% had a moderate stroke (NIHSS, 5 to 15 ) and $16.3 \%$ suffered from a severe stroke (NIHSS >15). At 3 months, $23.9 \%$ of the included patients had an unfavorable outcome (10.8\% had mRS 3, 8.7\% mRS 4, 1.1\% mRS 5, and $3.3 \%$ mRS 6 ). Vessel occlusion ( $95.4 \%$ vs. $42.8 \%, P<0.0001$ ), IS as first-ever CIE ( $100 \%$ vs. $0 \%, P=0.03)$, high admission NIHSS ( $15 \pm 6$ vs. $4.8 \pm 5.7, P<0.0001)$ and low admission DWI-ASPECTS (4.0 \pm 2.1 vs. $7.7 \pm 1.6, P<0.0001)$ were associated with 3 -month unfavorable outcomes. At the final follow-up visit, 92.4\% achieved a favorable outcome (mRS 0-2). 
Table 1. Baseline characteristics of the patients

\begin{tabular}{|c|c|}
\hline Characteristic & All patients $(n=92)$ \\
\hline Age at first-ever CIE (yr) & $49.3 \pm 9.7(26-72)$ \\
\hline Age at inclusion (yr) & $49.8 \pm 9.9(27-73)$ \\
\hline First-ever CIE to last visit (mo) & $53.2 \pm 33.6(0.1-179)$ \\
\hline Study enrollment to last visit (mo) & $50.5 \pm 29.6(0.1-135)$ \\
\hline Male sex & $40(43.5)$ \\
\hline Vascular risk factor & 40 (43.5) \\
\hline Arterial hypertension & $19(20.6)$ \\
\hline Diabetes & $3(3.3)$ \\
\hline Hyperlipidemia & $9(9.8)$ \\
\hline Smoking & $19(20.6)$ \\
\hline Left symptomatic CaW & $48(52.2)$ \\
\hline Admission NIHSS & $7.7 \pm 7.4(0-27)$ \\
\hline Admission DWI-ASPECTS* & $7.2 \pm 2.5(0-10)$ \\
\hline Modified Rankin Scale score 0-2 at 3 months & $70(76.1)$ \\
\hline Modified Rankin Scale score $0-2$ at final follow-up visit & $85(92.4)$ \\
\hline \multicolumn{2}{|l|}{ Index CIE } \\
\hline TIA & $13(14.1)$ \\
\hline Acute ischemic stroke & $79(85.9)$ \\
\hline Superficial MCA infarction & $42(45.7)$ \\
\hline Deep MCA infarction & $10(10.9)$ \\
\hline Total MCA infarction & $22(23.9)$ \\
\hline Fetal disposition PCA infarction & $3(3.3)$ \\
\hline Retinal infarction & $2(2.2)$ \\
\hline Vessel occlusion & $51(55.4)$ \\
\hline Anterior cerebral artery & $2(2.2)$ \\
\hline M1 MCA & $30(32.6)$ \\
\hline M2 MCA & $13(14.1)$ \\
\hline M3 MCA & $2(2.2)$ \\
\hline Internal cerebral artery & $4(4.3)$ \\
\hline Silent cerebral infarcts ipsilateral to CIE & $7(7.6)$ \\
\hline ECST stenosis (\%) & $36 \pm 14(10-90)$ \\
\hline ESCT stenosis $>35 \%$ & $42(45.6)$ \\
\hline Presence of contralateral CaW & $42(45.6)$ \\
\hline Carotid surgery or stenting & $23(25)$ \\
\hline
\end{tabular}

Values are presented as mean \pm standard deviation (range) or number (\%).

$\mathrm{CIE}$, cerebral ischemic event; CaW, carotid web; NIHSS, National Institutes of Health Stroke Scale; DWI-ASPECTS, diffusion-weighted imaging Alberta Stroke Program Early CT score; TIA, transient ischemic attack; MCA, middle cerebral artery; PCA, posterior cerebral artery; ECST, European Carotid Surgery Trial.

*Data derived from patients with MCA infarction $(n=74)$.

\section{Imaging characteristics of first-ever CIE associat- ed with a $\mathrm{CaW}$}

At inclusion, 13 patients experienced a TIA as first-ever CIE and two patients suffered from a central retina artery infarction without any recent lesion on admission MRI. Of the 92 patients included, 42 (45.7\%) had superficial MCA territory infarction (the most frequent infarction pattern) as their first-ever CIEs, and 51 (55.4\%) had large vessel or branch occlusion. In patients with MCA infarction, the mean DWI-ASPECTS score was $7.2 \pm 2.5$ (range, 0 to 10 ) and $25.8 \%$ experienced a very large stroke (DWI-ASPECTS $\leq 5$ ).

$\mathrm{SCl}$ ipsilateral to the index $\mathrm{CIE}$ were found in seven patients 
Table 2. Characteristics of patients treated with medical therapy alone and those with surgery or stenting

\begin{tabular}{|c|c|c|c|}
\hline Characteristic & Medical treatment group $(n=69)$ & Surgery/stenting treatment group $(n=23)$ & $P$ \\
\hline Age at first-ever CIE (yr) & $50.4 \pm 9.5(26-72)$ & $45.9 \pm 9.7(28-71)$ & 0.020 \\
\hline Age at inclusion (yr) & $51.1 \pm 9.9(27-73)$ & $46.2 \pm 9.7(29-72)$ & 0.020 \\
\hline First-ever CIE to last visit (mo) & $53.1 \pm 34.6(1-177)$ & $55.6 \pm 28.8(12-125)$ & 0.560 \\
\hline Study enrollment to last visit (mo) & $49.7 \pm 29.5(1-136)$ & $54.3 \pm 30.1(3-125)$ & 0.440 \\
\hline Male sex & $29(42.0)$ & $11(47.8)$ & 0.800 \\
\hline Vascular risk factor & $34(49.3)$ & $6(26.1)$ & 0.090 \\
\hline Arterial hypertension & $17(24.6)$ & $2(8.7)$ & 0.180 \\
\hline Diabetes & $3(4.4)$ & $0(0)$ & 0.730 \\
\hline Hyperlipidemia & $8(11.6)$ & $1(4.4)$ & 0.500 \\
\hline Smoking & $16(23.2)$ & $3(13.0)$ & 0.450 \\
\hline TIA as index $\mathrm{CIE}$ & $12(17.4)$ & $1(4.4)$ & 0.170 \\
\hline Admission NIHSS & $7.5 \pm 7.5(0-27)$ & $8.1 \pm 7.2(0-24)$ & 0.600 \\
\hline Admission DWI-ASPECTS* & $7.4 \pm 2.5(0-10)$ & $6.5 \pm 2.2(1-10)$ & 0.060 \\
\hline Vessel occlusion & $32(46.4)$ & $19(82.6)$ & 0.005 \\
\hline Silent cerebral infarction on first imaging & $5(7.3)$ & $2(8.7)$ & 0.990 \\
\hline $\mathrm{mRS}$ score $0-2$ at 3 months & $52(75.4)$ & $18(78.3)$ & 0.990 \\
\hline $\mathrm{mRS}$ score $0-2$ at last follow-up visit & $63(91.3)$ & $22(95.7)$ & 0.820 \\
\hline ECST stenosis (\%) & $34 \pm 0.14(10-90)$ & $43 \pm 13(19-74)$ & 0.002 \\
\hline Presence of contralateral CaW & $31(44.9)$ & $11(47.8)$ & 0.990 \\
\hline Ipsilateral CIE recurrence & $12(17.4)$ & $7(30.4)^{+}$ & 0.290 \\
\hline
\end{tabular}

Values are presented as mean \pm standard deviation (range) or number (\%).

CIE, cerebral ischemic event; TIA, transient ischemic attack; NIHSS, National Institutes of Health Stroke Scale; DWI-ASPECTS, diffusion-weighted imaging AIberta Stroke Program Early CT score; mRS, modified Rankin Scale; ECST, European Carotid Surgery Trial; CaW, carotid web.

${ }^{*}$ Data derived from patients with middle cerebral artery infarction $(n=74) ;{ }^{+}$Recurrent ClEs occurred before surgery or stenting.

(7.6\%) while one patient with bilateral CaW showed SCI contralateral to the index CIE. All SCls were cortical or cortico-subcortical lesions.

\section{Acute and long-term treatments}

Thirty-three patients (35.9\%) received intravenous thrombolysis, six $(6.5 \%)$ were treated with mechanical thrombectomy and three (3.3\%) underwent decompressive hemicraniectomy. At the end of follow-up, preventive therapy was as follow: antiplatelet treatment in $52(56.5 \%)$ patients, oral anticoagulation treatment in $12(13 \%)$, no specific preventive treatment in five (5.5\%), carotid artery surgery in $21(22.8 \%)$, and carotid artery stenting in two (2.2\%). All patients treated with carotid surgery or stenting received long-term antithrombotic therapy with aspirin $(n=21)$ or vitamin $K$ antagonist $(n=2)$ that was ongoing at the end of follow-up. Comparisons between the surgery/stenting group ( $n=23)$ and the medical treatment group $(n=69)$ are summarized in Table 2. Compared to patients managed with medical therapy alone, patients who received surgery or stenting were younger at the time of first-ever CIE $(45.9 \pm 9.7$ years vs. $50.4 \pm 9.5$ years, $P=0.02$ ), were more likely to have vessel occlusion ( $82.6 \%$ vs. $46.7 \%, P=0.005$ ), and had more severe CaW stenosis ( $43 \% \pm 13 \%$ vs. $34 \% \pm 14 \%, P=0.002)$.

\section{Characteristics of symptomatic CaW}

In most patients (97\%), CaW was located on the posterior and lateral wall of the carotid bulb. According to the ECST method, the mean symptomatic carotid bulb stenosis was $36 \% \pm 14 \%$. Of 21 surgically resected webs, 14 specimens underwent pathological examinations. The web lesion showed a thin translucent intraluminal diaphragm on macroscopic inspection and the histological examination revealed focal intimal hyperplasia that minimally involved the media layer. A contralateral asymptomatic CaW was diagnosed in 42 patients (46.5\%). In these patients, the stenosis degree was less severe in the asymptomatic CaWs than in the symptomatic CaWs $(30 \% \pm 13 \%$ vs. $37 \% \pm 15 \%$, $P=0.03)$.

\section{Recurrences}

Nineteen (20.7\%) out of 92 patients experienced a recurrent 
Table 3. Characteristics of patients with recurrent $\mathrm{CIE}$ and those without

\begin{tabular}{|c|c|c|c|}
\hline Characteristic & No recurrence $(n=73)$ & Ipsilateral recurrence $(n=19)$ & $P$ \\
\hline Age at first-ever CIE (yr) & $49.3 \pm 9.6(26-72)$ & $49.1 \pm 10.6(36-71)$ & 0.640 \\
\hline Age at inclusion (yr) & $49.9 \pm 9.7(27-73)$ & $49.7 \pm 11.1(36-72)$ & 0.680 \\
\hline First-ever CIE to last visit (mo) & $50.1 \pm 28.7(0.1-127)$ & $66.4 \pm 46.6(6-179)$ & 0.270 \\
\hline Study enrollment to last visit (mo) & $49.9 \pm 28.4(0.1-127)$ & $54.5 \pm 34(6-135)$ & 0.750 \\
\hline Male sex & $27(37.0)$ & $13(68.4)$ & 0.020 \\
\hline Vascular risk factor & $32(43.8)$ & $8(42.1)$ & 0.990 \\
\hline Arterial hypertension & $16(21.9)$ & $3(15.8)$ & 0.780 \\
\hline Diabetes & $3(4.1)$ & $0(0)$ & 0.860 \\
\hline Hyperlipidemia & $5(6.8)$ & $4(2.1)$ & 0.150 \\
\hline Smoking & $14(19.2)$ & $5(2.6)$ & 0.710 \\
\hline $\mathrm{TIA}$ as index $\mathrm{CIE}$ & $11(15.1)$ & $2(10.5)$ & 0.890 \\
\hline Admission NIHSS & $8 \pm 7.7(0-27)$ & $6.5 \pm 6.2(0-19)$ & 0.570 \\
\hline Admission DWI-ASPECTS* & $7.2 \pm 2.6(0-10)$ & $7.4 \pm 1.7(5-10)$ & 0.940 \\
\hline Vessel occlusion & $38(52.1)$ & $13(68.4)$ & 0.300 \\
\hline Silent cerebral infarction on first imaging & $2(2.7)$ & $5(26.3)$ & 0.003 \\
\hline $\mathrm{mRS}$ score $0-2$ at 3 months & $54(74.0)$ & $16(84.2)$ & 0.520 \\
\hline mRS score $0-2$ at last follow-up visit & $66(90.4)$ & $19(100)$ & 0.350 \\
\hline ECST stenosis (\%) & $36 \pm 14(10-90)$ & $38 \pm 13(15-63)$ & 0.370 \\
\hline Presence of contralateral CaW & $34(46.6)$ & $8(42.1)$ & 0.920 \\
\hline Carotid surgery or stenting & 16 (21.9) & $7(36.8)^{+}$ & 0.290 \\
\hline
\end{tabular}

Values are presented as mean \pm standard deviation (range) or number (\%).

CIE, cerebral ischemic event; TIA, transient ischemic attack; NIHSS, National Institutes of Health Stroke Scale; DWI-ASPECTS, diffusion-weighted imaging AIberta Stroke Program Early CT score; mRS, modified Rankin Scale; ECST, European Carotid Surgery Trial; CaW, Carotid web.

${ }^{*}$ Data derived from patients with middle cerebral artery infarction $(n=74) ;{ }^{\dagger}$ Recurrent CIEs occurred before surgery or stenting.

CIE (16 ISs and three TIAs) during the follow-up period. Characteristics of patients with and without $\mathrm{CIE}$ recurrence are detailed and compared in Table 3. Male sex (68.4\% vs. $37.0 \%$, $P=0.02)$ and presence of ipsilateral $\mathrm{SCl}(26.3 \%$ vs. $2.7 \%$, $P=0.003$ ) were significantly associated with recurrences on univariable analysis.

All recurrences were ipsilateral to the first-ever CIE. At the time of recurrent $\mathrm{CIE}, 16$ patients were taking antiplatelet monotherapy, two were on oral anticoagulant therapy, and one did not have any anti-thrombotic therapies. In 42 patients with bilateral CaWs, no CIE recurrence was associated with asymptomatic CaW. The median follow-up time of asymptomatic CaW (inclusion to last visit delay) was 44.8 months (IQR, 36.1 to 66).

In patients who received carotid surgery or stenting for symptomatic CaW, no recurrence occurred after the intervention. In these patients, the median interval from the first-ever CIE to surgery or stenting was 7.5 months (IQR, 4.6 to 12.5) and the median follow-up time after surgery or stenting was 39.8 months (IQR, 27.6 to 72.4). The median follow-up time of patients under medical treatment alone (for patients without
Kaplan-Meier curves for time to recurrent CIE and IS under medical treatment

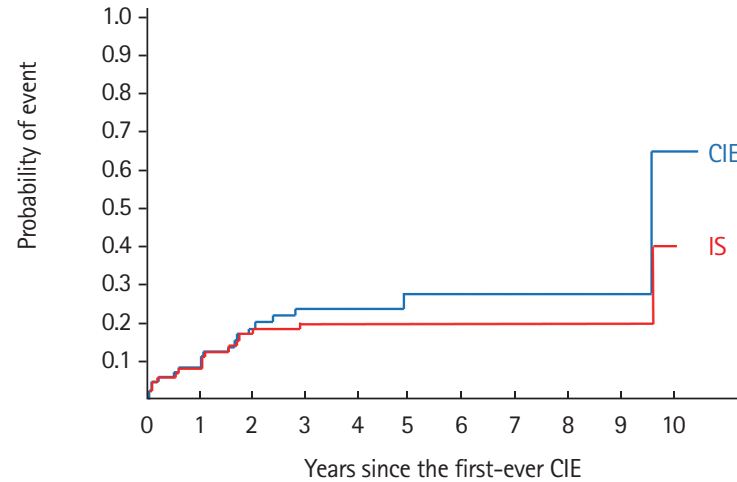

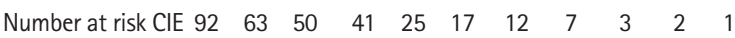
Number at risk IS $92 \begin{array}{llllllllll}63 & 51 & 43 & 27 & 20 & 15 & 9 & 4 & 3 & 2\end{array}$

Figure 1. Kaplan-Meier curves of cumulative rates of cerebral ischemic event and ischemic stroke recurrences in symptomatic carotid web patients medically treated. CIE, cerebral ischemic event; IS, ischemic stroke.

surgery or stenting, the whole follow-up period was considered, 
Table 4. Predictors for recurrent cerebrovascular ischemic event in patients under medical treatment: unadjusted and adjusted analyses

\begin{tabular}{|c|c|c|c|c|}
\hline \multirow{2}{*}{ Characteristic } & \multicolumn{2}{|c|}{ Unadjusted } & \multicolumn{2}{|c|}{ Adjusted } \\
\hline & $\mathrm{HR}(95 \% \mathrm{Cl})$ & $P$ & HR (95\% Cl) & $P$ \\
\hline Age at first-ever CIE (yr) & $0.99(0.94-1.04)$ & 0.650 & - & - \\
\hline Age at inclusion (yr) & $0.99(0.95-1.04)$ & 0.720 & - & - \\
\hline First-ever CIE to last visit (mo) & $1.00(0.99-1.01)$ & 0.910 & - & - \\
\hline Study enrollment to last visit (mo) & $0.99(0.98-1.01)$ & 0.490 & - & - \\
\hline Male sex & $2.75(1.03-7.33)$ & 0.040 & $2.27(0.83-6.16)$ & 0.110 \\
\hline Vascular risk factor & $0.82(0.34-2.09)$ & 0.710 & - & - \\
\hline Arterial hypertension & $1.67(0.48-5.81)$ & 0.410 & - & - \\
\hline Diabetes & $1.60(0.21-12.13)$ & 0.640 & - & - \\
\hline Hyperlipidemia & $2.60(0.84-7.98)$ & 0.090 & - & - \\
\hline Smoking & $1.13(0.40-3.16)$ & 0.820 & - & - \\
\hline TIA as index $\mathrm{CIE}$ & $0.60(0.14-2.63)$ & 0.500 & - & - \\
\hline Admission NIHSS & $1.00(0.93-1.06)$ & 0.950 & - & - \\
\hline Admission DWI-ASPECTS* & $1.01(0.83-1.22)$ & 0.930 & - & - \\
\hline Vessel occlusion & $2.19(0.82-5.85)$ & 0.120 & - & - \\
\hline Silent cerebral infarction on first imaging & $8.44(2.94-24.24)$ & $<0.001$ & $6.99(2.40-20.40)$ & 0.004 \\
\hline ECST stenosis (\%) & $5.71(0.37-86.90)$ & 0.210 & - & - \\
\hline Presence of contralateral CaW & $0.64(0.25-1.64)$ & 0.350 & - & - \\
\hline
\end{tabular}

$\mathrm{HR}$, hazard ratio; $\mathrm{Cl}$, confidence interval; $\mathrm{CIE}$, cerebral ischemic event; TIA, transient ischemic attack; NIHSS, National Institutes of Health Stroke Scale; DWI-ASPECTS, diffusion-weighted imaging Alberta Stroke Program Early CT score; ECST, European Carotid Surgery Trial; CaW, carotid web.

${ }^{*}$ Data derived from patients with middle cerebral artery infarction $(n=74)$.
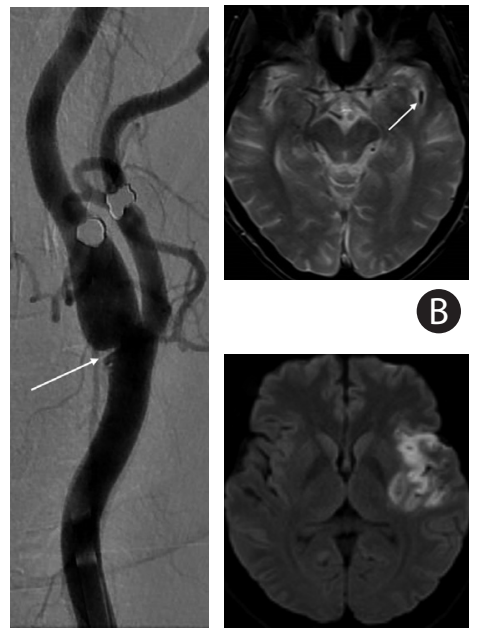

A
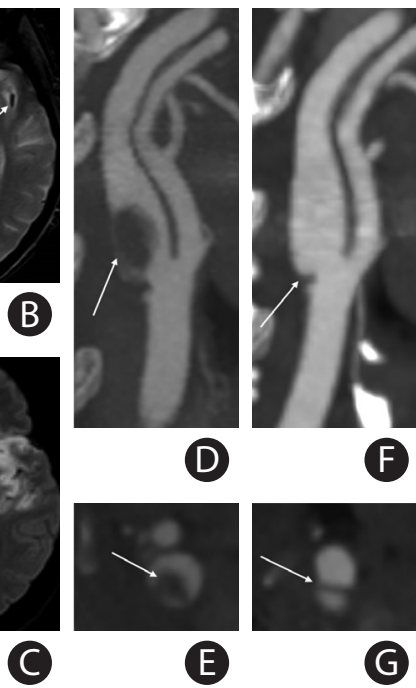

(D)
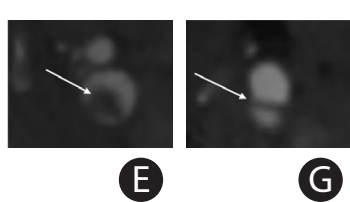

G

Figure 2. Illustrative case who experienced a recurrent ischemic stroke in the left MCA territory under antiplatelet treatment. Carotid web (CaW) (white arrow) on digital subtraction angiography obtained at the index event of transient right-side hemiplegia in a 42-year-old woman (A). The patient was treated with aspirin (160 $\mathrm{mg} /$ day). Eleven months later, the patient developed right hemiplegia and aphasia. Brain magnetic resonance imaging gradient echo sequences showed a low signal intensity lesion (white arrow) in the left middle cerebral artery (MCA) (B), and diffusion weighted imaging confirmed acute ischemic stroke in the left MCA territory (C). Sagittal (D) and axial (E) views of computed tomography angiography (CTA) showed a mural thrombus superimposed to the previously identified CaW (white arrows). Follow-up CTA obtained after anticoagulation for 2 weeks showed disappearance of the mural thrombus (white arrows): sagittal (F) and axial (G) plane. and for patients who received surgery or stenting, the period from the inclusion to the day before surgery or stenting was considered as the follow-up duration of medical treatment alone) was 36 months (IOR, 11 to 58).

In all patients, the annual recurrent CIE rate was 5.4\%/year. When we analyzed the recurrent CIE rate for the follow-up period under medical treatment alone, it was 6.9\%/year. For the cumulative CIE rate during the follow-up under medical treatment alone, it was $4.4 \%$ at 30 -day, $10.8 \%$ at 1 -year, $19.8 \%$ at 2-year, 23.2\% at 3-year, 27.3\% at 5-year, and 63.6\% at 10-year (Figure 1 and Supplementary Table 1). The cumulative CIE rates for the whole follow-up period and for subgroups according to sex and $\mathrm{SCl}$ presence are detailed in Supplementary Table 1. In multivariable Cox proportional regression analysis, the presence of $\mathrm{SCl}$ was the only independent risk factor of $\mathrm{CIE}$ recurrence ( $\mathrm{HR}$, 6.99; $95 \% \mathrm{Cl}, 2.4$ to $20.4 ; P=0.004$ ) (Table 4). A case of recurrent $\mathrm{CIE}$ is illustrated in Figure 2.

\section{Discussion}

We report a large series of symptomatic CaW and provide prospective data regarding the recurrent rate of IS or TIA. Our main findings are: (1) Despite preventive antithrombotic treatment, 
symptomatic CaWs were associated with a high risk of CIE recurrences that reached $10.8 \%$ at 1-year, $19.8 \%$ at 2-year, and $27.3 \%$ at 5-year. (2) $\mathrm{SCl}$ ipsilateral to the symptomatic CaW was an independent risk factor of $\mathrm{CIE}$ recurrence $(\mathrm{HR}, 6.99$; $95 \% \mathrm{Cl}, 2.4$ to $20.4 ; P=0.004$ ). (3) Surgery or stenting of CaW seems to be efficient as no recurrence was identified after the intervention with a median follow-up time of 39.8 months (IQR, 27.6 to 72.4). (4) Recurrent CIEs were exclusively associated with symptomatic $\mathrm{CaW}$, and there was no recurrent CIE related to asymptomatic CaW.

Association between CaW and ipsilateral stroke recurrence has been described since the first case reports. ${ }^{1.18}$ Three small case series showed that symptomatic CaW patients had a high risk of stroke recurrences ranging from 25\% to $71.4 \%$. $^{3,11,13}$ In accordance with our findings, recurrent strokes occurred under antiplatelet treatment. Whether the index stroke was a first-ever CIE was not clear in these studies. A recent systematic literature review found a 56\% rate of recurrence among patients medically treated..$^{19}$ However, the analysis was performed on small series and single observations of interest and the recurrent rate may be overestimated. In our study, the curve for cumulative incidence of $\mathrm{CIE}$ is steepest in the first 2 years after the first-ever CIE. Subsequently, the ascent appears less steep between 2 and 5 years. Although CaW is still listed as a cause of $\mathrm{CIS}_{1}^{20}$ we showed that the recurrent stroke or TIA risk with symptomatic CaW was much higher than those reported in young patients with usual $\mathrm{CIS}$ for whom the cumulative 5-year rate has been estimated at $8.2 \% .{ }^{21}$ In a recent randomized trial for patients with embolic stroke of undetermined source, the annual recurrent stroke rate under aspirin was $4.8 \%{ }^{22}$ Patients medically treated for CIS associated with patent foramen ovale also had a lower recurrent risk estimated at 1.17\% per year. ${ }^{23}$ In our cohort, the 23 patients treated with carotid surgery or stenting were free of recurrence after a median follow-up of 39 months. Surgery has been proposed as invasive treatment option, ${ }^{3}$ and small series of carotid endarterectomy in symptomatic CaW have reported data for acceptable safety and efficacy after a median follow-up around 12 months. ${ }^{3,13,24-26}$ Endovascular procedure has also been reported as safe, ${ }^{27}$ and a recent study ${ }^{28}$ showed that the procedure performed in 24 symptomatic CaW patients was effective to prevent recurrence after a median clinical follow-up of 12 months. Although no randomized data are available, invasive treatment such as surgery or stenting seems now increasingly proposed as secondary preventive strategy. ${ }^{29}$ In the present study, patients treated with surgery or stenting had a higher rate of vessel occlusion and a more severe stenosis related to CaW than medically treated patients. Physicians and patients may have been more convinced that $\mathrm{CaW}$ was responsible for $\mathrm{CIE}$ when the lesion was more prominent and associated with artery occlusion. No recurrence was recorded in the contralateral carotid territory even in patients with bilateral CaW. Our findings suggest that surgery or stenting is not recommended for asymptomatic CaW.

We found that the risk of $\mathrm{CIE}$ recurrences was 2.7 times higher in males compared to females. This result was not supported by other studies that usually reported no difference ${ }^{30}$ in stroke recurrence between males and females or a slightly higher risk in females. ${ }^{31} \mathrm{SCl}$ ipsilateral to the first-ever $\mathrm{CIE}$, was detected in $7.6 \%$ of our cohort and constituted a strong predictor of recurrence ( $\mathrm{HR}, 8.4 ; 95 \% \mathrm{Cl}, 2.9$ to $24 ; P<0.001)$. The presence of $\mathrm{SCl}$ might indicate a high embolic potential of the CaW lesion.

This study has several limitations. Although all cases showed classic imaging characteristics of CaW, the lesion was histologically proven in a minority of patients. The cohort study was implemented in the Caribbean area in 2008 and thereby focused only on Afro-Caribbean patients. Our findings should be interpreted with caution when applied to other ethnic groups. Recurrent CIEs were often reported by patients, which might underestimate the recurrent rate. A small number of patients had $\mathrm{SCl}(n=7)$ and the association with recurrent risk should be considered with caution. We obtained no data on adherence to antithrombotic therapies, particularly for long-term antiplatelet treatment. Secondary stroke prevention therapies other than antithrombotic therapy was not systemically captured and therapeutic strategies differed overtime and across the participating centers. Thus, the different efficiency for preventing recurrent stroke between medical treatment alone and invasive treatment should be interpreted with caution. However, the baseline characteristics of patients without and those with surgery or stenting were generally comparable. Given that patients who received surgery or stenting group had more severe $\mathrm{CaW}$ stenosis and more vessel occlusions, these patients might be at higher risk of recurrent CIE without the intervention. The strengths of this study are the prospective identification of CIE recurrences with a large cohort of symptomatic CaW and the long follow-up period. Additionally, symptomatic CaW is now increasingly treated with stenting or surgery and data on recurrence under medical treatment might be hardly available in the future.

\section{Conclusions}

This study provides key data on IS and TIA recurrences after a first-ever CIE associated with symptomatic CaW. Recurrent ISs or TIAs occurred in 19.8\% at 2-year and 27.3\% at 5-year despite antithrombotic treatment. $\mathrm{SCl}$ ipsilateral to symptomatic 
CaW was the only predictor of recurrences. Given the high recurrent risk under medical treatment alone, CaW should be systematically investigated in patients with CIS. In our cohort, invasive treatments such as surgery excision and endovascular stenting were safe and effective to prevent recurrences. Our data support further clinical trials that could compare medical and interventional therapies in patients with $\mathrm{CIS}$ and $\mathrm{CaW}$.

\section{Supplementary materials}

Supplementary materials related to this article can be found online at https://doi.org/10.5853/jos.2020.05225.

\section{Disclosure}

The authors have no financial conflicts of interest.

\section{References}

1. Wirth FP, Miller WA, Russell AP. Atypical fibromuscular hyperplasia. Report of two cases. J Neurosurg 1981;54:685689.

2. Morgenlander JC, Goldstein LB. Recurrent transient ischemic attacks and stroke in association with an internal carotid artery web. Stroke 1991;22:94-98.

3. Joux J, Chausson N, Jeannin S, Saint-Vil M, Mejdoubi M, Hennequin JL, et al. Carotid-bulb atypical fibromuscular dysplasia in young Afro-Caribbean patients with stroke. Stroke 2014;45:3711-3713.

4. Joux J, Boulanger $M$, Jeannin $S$, Chausson $N$, Hennequin JL,

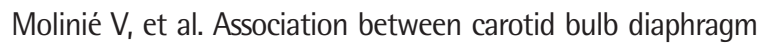
and ischemic stroke in young Afro-Caribbean patients: a population-based case-control study. Stroke 2016;47:2641-2644.

5. Coutinho JM, Derkatch S, Potvin AR, Tomlinson G, Casaubon $\mathrm{LK}$, Silver $\mathrm{FL}$, et al. Carotid artery web and ischemic stroke: a case-control study. Neurology 2017;88:65-69.

6. Sajedi PI, Gonzalez JN, Cronin CA, Kouo T, Steven A, Zhuo J, et al. Carotid bulb webs as a cause of "cryptogenic" ischemic stroke. AJNR Am J Neuroradiol 2017;38:1399-1404.

7. Kim SJ, Allen JW, Bouslama M, Nahab F, Frankel MR, Nogueira RG, et al. Carotid webs in cryptogenic ischemic strokes: a matched case-control study. J Stroke Cerebrovasc Dis 2019;28:104402.

8. Kubis N, Von Langsdorff D, Petitjean C, Brouland JP, Guichard JP, Chapot R, et al. Thrombotic carotid megabulb: fibromuscular dysplasia, septae, and ischemic stroke. Neurology 1999;52:883-886.

9. Olindo S, Marnat G, Chausson N, Turpinat C, Smadja D, Gail- lard N. Carotid webs associated with ischemic stroke. Updated general review and research directions. Rev Neurol (Paris) 2021 Jan 14 [Epub]. https://doi.org/10.1016/j.neurol.2020.09.007.

10. Compagne KCJ, Dilba K, Postema EJ, van Es ACGM, Emmer $B J$, Majoie CBLM, et al. Flow patterns in carotid webs: a patient-based computational fluid dynamics study. AJNR Am J Neuroradiol 2019;40:703-708.

11. Haussen DC, Grossberg JA, Bouslama M, Pradilla G, Belagaje $S$, Bianchi N, et al. Carotid web (intimal fibromuscular dysplasia) has high stroke recurrence risk and is amenable to stenting. Stroke 2017;48:3134-3137.

12. Compagne KCJ, van Es ACGM, Berkhemer OA, Borst J, Roos YBWEM, van Oostenbrugge RJ, et al. Prevalence of carotid web in patients with acute intracranial stroke due to intracranial large vessel occlusion. Radiology 2018;286:10001007.

13. Choi PM, Singh D, Trivedi A, Qazi E, George D, Wong J, et al. Carotid webs and recurrent ischemic strokes in the era of CT angiography. AJNR Am J Neuroradiol 2015;36:2134-2139.

14. Barber PA, Demchuk AM, Zhang J, Buchan AM. Validity and reliability of a quantitative computed tomography score in predicting outcome of hyperacute stroke before thrombolytic therapy. ASPECTS Study Group. Alberta Stroke Programme Early CT Score. Lancet 2000;355:1670-1674.

15. Sacco RL, Kasner SE, Broderick JP, Caplan LR, Connors JJ, Culebras A, et al. An updated definition of stroke for the 21st century: a statement for healthcare professionals from the American Heart Association/American Stroke Association. Stroke 2013;44:2064-2089.

16. Madaelil TP, Grossberg JA, Nogueira RG, Anderson A, Barreira $\mathrm{C}$, Frankel $\mathrm{M}$, et al. Multimodality imaging in carotid web. Front Neurol 2019;10:220.

17. European Carotid Surgery Trialists' Collaborative Group. MRC European Carotid Surgery Trial: interim results for symptomatic patients with severe (70-99\%) or with mild (0-29\%) carotid stenosis. Lancet 1991;337:1235-1243.

18. So EL, Toole JF, Moody DM, Challa VR. Cerebral embolism from septal fibromuscular dysplasia of the common carotid artery. Ann Neurol 1979;6:75-78.

19. Zhang AJ, Dhruv P, Choi P, Bakker C, Koffel J, Anderson D, et al. A systematic literature review of patients with carotid web and acute ischemic stroke. Stroke 2018;49:2872-2876.

20. Mac Grory B, Flood SP, Apostolidou E, Yaghi S. Cryptogenic stroke: diagnostic workup and management. Curr Treat Options Cardiovasc Med 2019;21:77.

21. Putaala J, Haapaniemi E, Metso AJ, Metso TM, Artto V, Kaste $M$, et al. Recurrent ischemic events in young adults after first-ever ischemic stroke. Ann Neurol 2010;68:661-671. 
22. Diener $H C$, Sacco RL, Easton JD, Granger CB, Bernstein RA, Uchiyama $S$, et al. Dabigatran for prevention of stroke after embolic stroke of undetermined source. N Engl J Med 2019; 380:1906-1917.

23. Ahmad Y, Howard JP, Arnold A, Shin MS, Cook C, Petraco R, et al. Patent foramen ovale closure vs. medical therapy for cryptogenic stroke: a meta-analysis of randomized controlled trials. Eur Heart J 2018;39:1638-1649.

24. Verscheure D, Fabre D, Smadja D, Fadel E. Symptomatic carotid-bulb atypical fibromuscular dysplasia. Ann Vasc Surg 2017;43:310.

25. Pereira BJA, Batista UC, Tosello RT, Ströher IN, Baeta $A M_{1}$ Piske RL. Web vessels: literature review and neurointerventional management. World Neurosurg 2018;110:e907-e916.

26. Multon $S$, Denier $C$, Charbonneau $P$, Sarov $M$, Boulate $D_{1}$

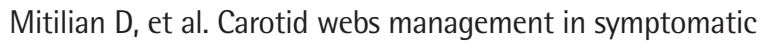
patients. J Vasc Surg 2021;73:1290-1297.
27. Brinjikji W, Agid R, Pereira VM. Carotid stenting for treatment of symptomatic carotid webs: a single-center case series. Interv Neurol 2018;7:233-240.

28. Haussen DC, Grossberg JA, Koch S, Malik A, Yavagal D, Gory $B$, et al. Multicenter experience with stenting for symptomatic carotid web. Interv Neurol 2018;7:413-418.

29. Wojcik K, Milburn J, Vidal G, Tarsia J, Steven A. Survey of current management practices for carotid webs. Ochsner J 2019;19:296-302.

30. Renoux C, Coulombe J, Li L, Ganesh A, Silver L, Rothwell PM, et al. Confounding by pre-morbid functional status in studies of apparent sex differences in severity and outcome of stroke. Stroke 2017;48:2731-2738.

31. Olindo $S$, Saint-Vil M, Jeannin S, Signate A, Edimonana-Kaptue $M$, Cabre $P$, et al. One-year disability, death and recurrence after first-ever stroke in a Black Afro-Caribbean population. Int J Stroke 2017;12:844-850. 
Supplementary Table 1. Cumulative recurrent CIE rates

\begin{tabular}{|c|c|c|c|c|c|c|}
\hline & \multicolumn{6}{|c|}{ Cumulative recurrent $\mathrm{CIE}$} \\
\hline & 30-Day & 1-Year & 2-Year & 3-Year & 5-Year & 10-Year \\
\hline CIE for the whole follow-up period & $4(4.4)$ & $9(10.1)$ & $15(17.7)$ & $17(20.4)$ & $18(23.5)$ & $19(49.0)$ \\
\hline $\mathrm{CIE}$ for the period under medical treatment alone & $4(4.4)$ & $9(10.8)$ & 15 (19.8) & $17(23.2)$ & $18(27.3)$ & 19 (63.6) \\
\hline \multicolumn{7}{|l|}{ According to sex } \\
\hline Males & $1(2.6)$ & $6(16.9)$ & $10(29.4)$ & $11(33.1)$ & $12(42.7)$ & $13(71.3)$ \\
\hline Females & $3(5.8)$ & $3(5.8)$ & $5(11.7)$ & $6(14.8)$ & $6(14.8)$ & $6(14.8)$ \\
\hline \multicolumn{7}{|l|}{ According to the presence of $\mathrm{SCl}$} \\
\hline $\mathrm{SCl}+$ & $2(31.4)$ & $3(48.6)$ & $5(82.9)$ & $5(82.9)$ & $5(82.9)$ & $5(82.9)$ \\
\hline $\mathrm{SCl}-$ & $2(2.4)$ & $6(8.1)$ & $10(14.4)$ & $12(18.1)$ & $13(22.4)$ & $14(61.2)$ \\
\hline IS for the period under medical treatment alone & $4(4.4)$ & $9(10.8)$ & 14 (18.3) & $15(20.0)$ & $15(20.0)$ & $16(40.0)$ \\
\hline
\end{tabular}

Values are presented as number (\%). All recurrent CIEs occurred ipsilateral to the first-ever CIE.

$\mathrm{CIE}$, cerebral ischemic event; $\mathrm{SCI}$, silent cerebral infarction; IS, ischemic stroke.
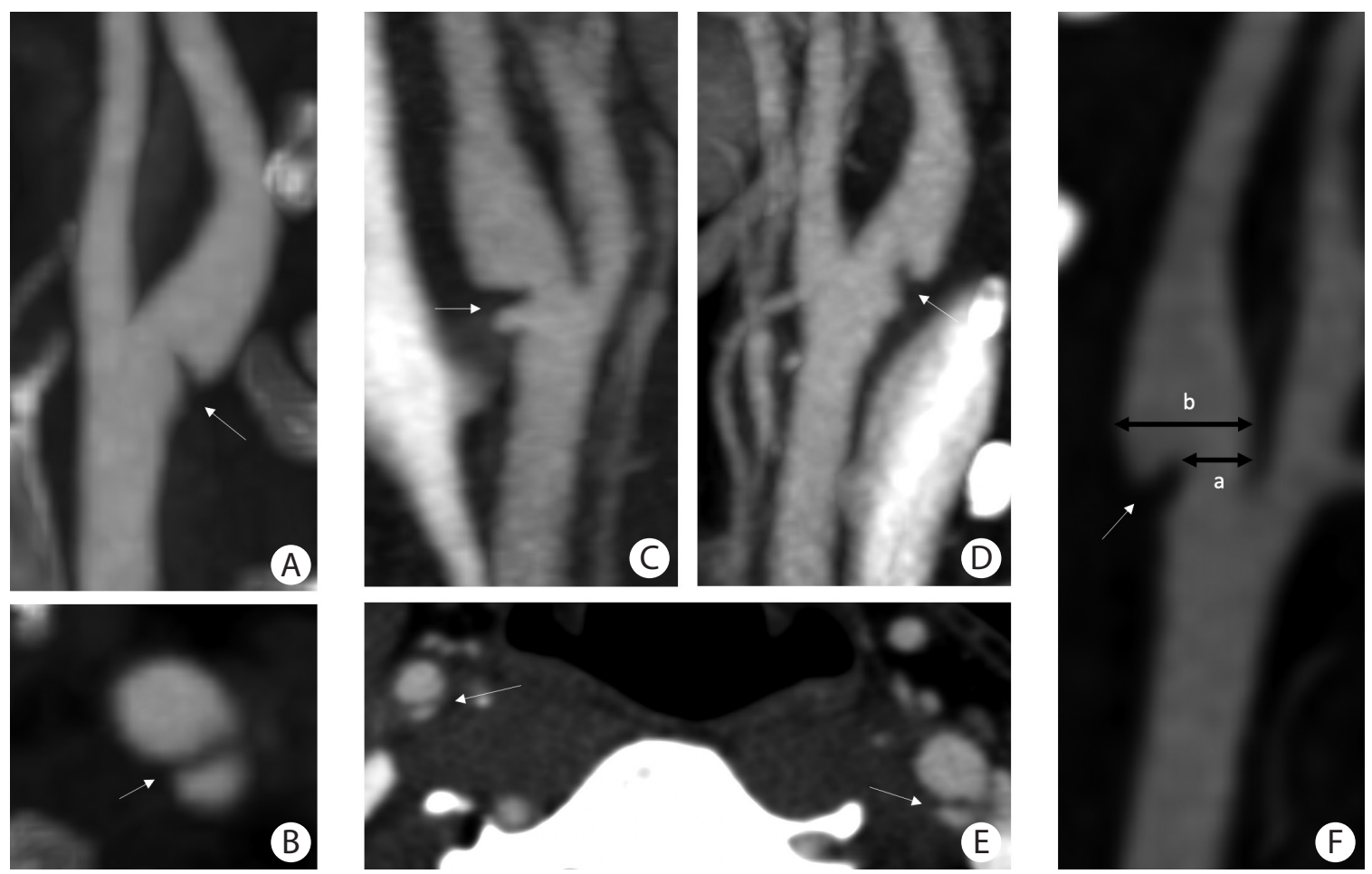

Supplementary Figure 1. Features of carotid web (CaW) on computed tomography angiography. CaW seen as a regular and thin shelf-like defect at the origin of the left carotid bulb (white arrow) on sagittal view (A), and the corresponding linear defect (white arrow) splitting the lumen artery on axial plane (B). Images of a patient with bilateral CaW (white arrows). Right asymptomatic CaW (C) and left symptomatic CaW (D) on sagittal view with the corresponding axial plane (E) that shows bilateral CaWs. Carotid bulb stenosis expressed in percentage was measured with the European Carotid Surgery Trial method: ([b a] / b) $\times 100$ (F). 